\title{
A Cross-Sectional Study of the Attitudes and Perceptions towards BiologicallyBased Therapies in the United Arab Emirates
}

\author{
Nawsheen Torabally ${ }^{1}$, Hananeh Abdolrahman Rahmanpoor*1,2 and Elhadi N Mahmoud ${ }^{1}$ \\ ${ }^{1}$ College of Pharmacy, Ajman University, U.A.E \\ ${ }^{2}$ Al Riyadh Pharmacy, Sharjah, U.A.E
}

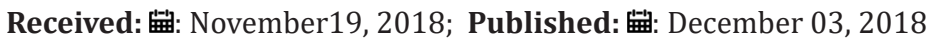

*Corresponding author: Hananeh Abdolrahman Rahmanpoor, College of Pharmacy, Ajman University, P.O.Box 346, U.A.E

\begin{abstract}
Biologically-Based Therapies (BBTs) are a subset of Complementary and Alternative Medicine (CAM) Therapies that fall into four main categories; diet therapy, herbal therapy, orthomolecular therapy and chelation therapy. BBTs are universally assumed to be safe and are widely used for the prevention and treatment of diseases via the use of nutrition, vitamins, minerals, herbal supplements, and chelating agents. A cross-sectional survey was carried out by Ajman University students on residents of the United Arab Emirates as to assess their knowledge and use of BBTs. This study aimed at determining whether healthcare providers were aware of their patient's use of BBTs and their safety profiles. Most respondents used BBTs, many of them lacked vital health information. Out of the respondents, $76 \%$ used herbal or other dietary supplements and $49 \%$ do not discus this with their healthcare providers. There is a significant amount of consumption coupled with insufficient awareness of BBTs in the UAE. Additionally, there is a lack of communication with healthcare providers and consumers of BBTs. As a result, consumers may potentially face inadvertent health complications depending on diet and other medications that are simultaneously being consumed.
\end{abstract}

Keywords: Biologically-Based Therapies (BBTs); Complementary \& Alternative Medicine (CAM); Knowledge; Attitude; Perception; Practices; Survey Questionnaire; Public Health

\section{Introduction}

Complementary and Alternative Medicine (CAM) is "a group of diverse medical and healthcare systems, practices, and products that are not presently considered to be part of conventional medicine" [1]. According to the National Center for Complementary and Alternative Medicine (NCCAM), CAM can be organized into the following categories: alternative medical systems, energy therapies manipulative and body based methods, mind-body interventions, and Biologically-Based therapies [2]. Due to the widespread use of CAM therapies, healthcare professionals are provided with continued medical education on the topic in addition to appropriate counseling techniques [3]. Biologically-Based Therapies (BBTs) are often used by patients in conjunction with conventional medicine and not simply as an alternative. BBTs consist of 4 types of therapies, namely: Diet, Herbal, Orthomolecular and Chelation Therapies [4-5].

Diet therapy is the practical application of nutrition for the prevention or treatment of a disease. This includes inclusion of certain beneficial foods into one's diet in order to promote wellness, while eliminating other foods to reduce harm. Herbal therapy involves the use of herbs for the purpose of treating common ailments and promoting general well being and is considered to be one of the earliest forms of healthcare. Orthomolecular therapy includes the use of vitamins, minerals, trace elements, electrolytes, fatty acids and amino acids for the purpose of treating specific conditions and/or preventing diseases. Chelation therapy involves the use of a binding agent that removes toxic metals or minerals, such as, lead, copper, iron, or calcium from the bloodstream [6]. In the past few decades, there has been an exponential growth in the use of diets, dietary supplements, and herbal compounds due to the assumption that their use is considered to be safe. It is assumed that these products promote general well-being through the prevention, correction and/or treatment of certain health conditions [7].

Many BBTs are available for purchase over the counter in the UAE. Communication with healthcare professionals and BBT consumers 
is vital because prescription drug interaction with herbal medicine is high [8]. It is essential for healthcare professionals to counsel patients on their use of BBTs and accurately assess their Dietary Reference Intake (DRI) for vitamins and minerals [9]. Despite a lack of clinical research on BBT's, most consumers use supplements after having self-diagnosed a suspected health condition [10]. The general risks of utilizing herbal/dietary supplements are adverse reactions and drug-supplement interactions. In some instances, substances may interact with prescription drugs and lead to potentially dangerous outcomes such as hemorrhaging [11]. Safety is an important aspect when considering alternative therapies. Regarding the risk of CAM treatments themselves, some are empirically deemed as safe, such as using ginger to treat nausea [12].

The US Food and Drug Administration (FDA) does not regulate Herbal/dietary supplements as regular drugs, because safety and efficacy data is not available for them [13]. The FDA typically intervenes if adverse reactions have been reported after the supplements have appeared on the market [14]. Dietary supplements may contain dangerous contaminants, toxic ingredients, or commercial drugs. Contaminated dietary supplements by microbes, pesticides, and heavy metals, led to adverse effects and resulted in removal of the supplements from the market by the FDA. Other reasons for recall include an absence or excess of the advertised active dietary ingredient [14]. Healthcare providers can more confidently provide adequate healthcare if they are familiar with the biochemical action of each component of the supplement on the body. It is necessary to understand how the individual supplement components interact with one another, how diseased state alters nutritional status, and how the prescription medication can affect nutrient deficiency [15]. The objective of this study was to determine the level of usage of BBTs among residents of the United Arab Emirates (U.A.E) and to assess their knowledge on the subject. Additionally, the study attempts to determine if healthcare providers are taking into consideration their patient's use of BBTs before prescribing or dispensing medications.

\section{Materials and Methods}

A cross-sectional survey was administered in the U.A.E focusing on the following variables: the number of people using BBTs in any form, public perception of BBTs, and if healthcare providers would consider any natural products or dietary supplements before prescribing/dispensing medications to patients. A crosssectional survey was chosen because it is the most thorough method of collecting a large quantity of information regarding behavior surrounding BBT consumption. This study did not aim in evaluating any temporal relationship with BBT consumption and perception of BBTs. A questionnaire comprising of 16 items, each with multiple selectable options was administered online using 'Google Docs'. Questionnaires were personally handed to students at Ajman University, located in the Emirate of Ajman. Additionally, the questionnaire was also available online. The collection of responses started from the $4^{\text {th }}$ of March 2011 until the $30^{\text {th }}$ April 2011. The survey questionnaire and results are included as supplementary files and are available upon request.
A total of 145 responses were obtained during that period of time. Random sampling was used to select the population in order to provide the most unbiased representative sample of the relatively large overall population. This study size was determined by the number of people that agreed to fill out the questionnaire and did so completely. The data analysis and descriptive statistics were found using Microsoft Excel 10. There was no exclusion criterion applied with participants. They represented all age groups, males and females, and it was inclusive of all ethnicities. All respondents consisted of U.A.E residents. The data collection occurred only at the aforementioned time, with no follow-up. The exposure to BBTs and the perceptions relating to their consumption was measured. The reasons for consumption, familiarity with BBT safety, and healthcare provider awareness of BBT consumption were outcomes measured in respondents that consumed BBTs.

The only quantitative variable measured in this study was age, and it was only analyzed using descriptive statistics with no focus on stratification by gender or BBT consumption. All 145 participants responded to each question on the survey. There may be some potential confounding variables in the data collection. Respondents may not be fully educated or aware of what exactly qualifies as a BBT. The data was self-responded, so there may be a degree of reporting bias as well, as a result of respondents not wanting to provide complete or true information. Attempts to alleviate sources of bias were done by informing participants of the definition of BBTs. Randomization of the sample was used as the primary method to counter confounders. Additionally, stratification of demographic variables, such as age and gender, was used to control for confounding.

\section{Results}

Out of the 145 respondents, $33 \%$ did not think it was important to mention it to their pharmacist that they were using a dietary supplement and $31 \%$ thought it was safe to use supplements at any time. Another 31\% answered that their pharmacist did not ask them about herbal or dietary supplements. It is the duty of the pharmacist to check with the patient if he is using any supplements before dispensing OTC drugs or filling a prescription and provide proper counseling. Out of the 110 respondents who use supplements, $72 \%$ of the respondents have spent more than United Arab Emirates Dirham 50 on supplements in the past year. To ensure that individuals are buying the right products at reasonable prices, especially in the case of herbal supplements, they need to have the right information about supplements. The amount of money spent on BBTs by respondents is also displayed in Table 2.

Table 1: The reason for using BBTs (more than one option can be selected, $\mathrm{n}=110)$.

\begin{tabular}{|c|c|}
\hline Reason for use & \# of individuals \\
\hline To correct vitamin or mineral deficiency & $37(34 \%)$ \\
\hline For minor ailments & $9(8 \%)$ \\
\hline To control blood sugar level & $6(5 \%)$ \\
\hline To lower blood cholesterol & $3(3 \%)$ \\
\hline For gastro-intestinal problems & $7(6 \%)$ \\
\hline
\end{tabular}




\begin{tabular}{|c|c|}
\hline For weight loss & $15(14 \%)$ \\
\hline As performance enhancer & $8(7 \%)$ \\
\hline As a boost for the brain & $18(16 \%)$ \\
\hline Other & $25(23 \%)$ \\
\hline
\end{tabular}

Table 2: Amount of money (AED) spent by respondents on supplements.

\begin{tabular}{|c|c|c|c|c|}
\hline < AED 50 & AED 50 - 99 & $\begin{array}{c}\text { AED 100 - } \\
\mathbf{1 4 9}\end{array}$ & $\begin{array}{c}\text { AED 150 - } \\
\mathbf{2 0 0}\end{array}$ & > AED 200 \\
\hline $31(28 \%)$ & $30(27 \%)$ & $14(13 \%)$ & $13(12 \%)$ & $22(20 \%)$ \\
\hline
\end{tabular}

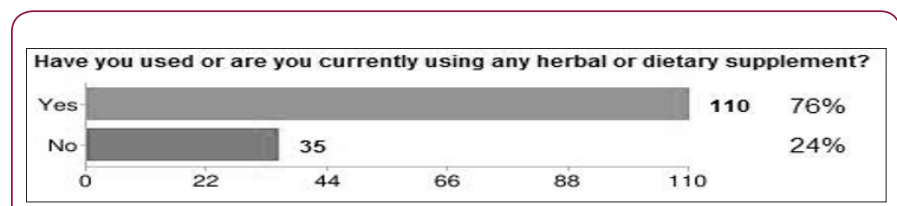

Figure 1: Percentage of respondents using herbal or dietary supplements.

Out of all respondents, 110 used or currently use BBT's, 45 (41\%) did so on occasion, 32 (29\%) used them regularly, 25 (23\%) experimented only once, and only 8 (7\%) consumed them diligently. Eighty-two percent of respondents were below the age of 30. It was determined that $30 \%$ acquired health information about herbal and/or dietary supplements from primary care providers, $15 \%$ from pharmacists, whereas $55 \%$ of people learned about supplements from other sources (such as friends, relatives, magazines, internet, nonmedical provider or other sources). Of this sample, $48 \%$ failed to inform their primary care providers about the use of supplements with only $12 \%$ having experienced an adverse reaction when asked about allergies. Most respondents did not believe it was necessary to inform their primary care providers on their use of BBT's. This subjective omission during a patient encounter may potentially result in various drug- interactions that may become problematic if not elucidated.

Out of the total sample size (145), 70\% were unaware of any possible food or drug interactions with herbal or dietary supplements. Concerning efficacy, $41 \%$ of all respondents used herbal/dietary supplements based on the assumption that it was as effective as conventional medicines, whereas $25 \%$ felt BBT's were superior. It is interesting to note that the most respondents bought their herbal/dietary supplements from pharmacies. Many felt that herbal/dietary supplements had fewer side effects than conventional medications. It may be inferred that this segment of the U.A.E population preferred herbal or nutritional supplements to conventional ones if given a choice. It is unclear, however, if respondents received appropriate counseling on the usage of supplements and whether those who bought their supplements from other sources had accurate information on BBT's.

\section{Discussion}

Optimal nutrition can be considered as a form of preventive medicine whereby BBT's are consumed for the promotion of good health such as in the developmental growth, the prevention of chronic health conditions, and treatment of immunodeficiency
[16]. It can be argued that there is an increased interest in herbal products based on the ease of availability. Pharmacists can play a key role in educating the public on healthy dietary regimens, accurate health information, and on the safe use of BBT's. It is therefore essential that they educate consumers on the possible adverse interactions between herbal supplements and prescription medications in addition to certain foods [17]. Our study concluded that $76 \%$ of all respondents used or currently use herbal products or other dietary supplements for the purpose of maintaining good health. More significantly, $48 \%$ of respondents who admitted to using herbal remedies did not disclose this information with their primary care physicians or pharmacists, which poses a problem for healthcare providers when it comes to discussing adverse drug interactions. Healthcare providers should take the appropriate steps during patient encounters and especially when prescribing or dispensing medications. Furthermore, many people are unaware of what symptoms they need to report to healthcare providers due to the overlap of side effects experienced using conventional medications.

The study has some inherent weaknesses. Despite Arabic being the official language of the U.A.E, the survey was conducted in English. This may be the source of some selection bias. Pharmacists are required to be proficient in Arabic but the resident population of the U.A.E is diverse and other languages were not surveyed, which may underrepresent the population. The resident population of the U.A.E is fluid as most visas have a two-year validity and results may vary if conducted on a regular basis. Future cross-sectional studies with multilingual options might provide a deeper insight on the beliefs of the population if conducted with fair regularity as able. Table 1 and Figure 1 show that there is a high amount of BBT consumption with other medication while respondents were not fully confident on BBT safety. This is significant because it shows that there is a degree of unfamiliarity that surround BBTs, yet there is still consumption. Currently, dietary or herbal supplements carry warning labels, nutritional information, and recommendations for consumers to consult with healthcare providers for further information on potential adverse reactions.

Pharmacists provide a crucial service in the delivery of care whereby they regularly counsel patients about the proper use of their prescription medications and based on this accessibility, they may be a patient's only immediate contact for healthcare information, especially as patient-physician interaction times are decreasing [18]. While the use of nutritional supplements is intended to enhance health, some BBT's have been found to interfere with prescribed medications [19]. This study can be used for a basis for more specifically focused studies regarding BBTs and consumption behavior. Similar studies can be conducted in regions with diverse populations in other Emirates or Gulf countries in order to determine the amount of familiarity consumers have with BBTs. From a public health perspective, pharmacists have the added responsibility of delivering health information. Preventive campaigns should not be limited to smoking and obesity, but also to the use of BBT's and potential harms that may result if not used properly. There is a plethora of BBT's in the U.A.E product market and the number of new products increases annually. 
Despite the intent to promote good health, information regarding BBT's remains highly limited and relatively unclear for many consumers. Further research concerning the efficacy of BBTs needs to be conducted so that sound regulations are established regarding their consumption and distribution. Overall, the use of CAM, which includes BBT's, has increased among patients that suffer from chronic medical illnesses and acute health conditions. Despite the wide array of BBTs, the prevalence and the scope of potential adverse effects with prescription drugs is unknown. Rapid changes in nutritional science and societal nutritional demands have led to challenges in the delivery of adequate healthcare. To compensate for these challenges, healthcare providers (such as dieticians, nurses and physicians) who are in direct contact with patients and consumers need to be actively involved in the dissemination of reliable information concerning BBT's and CAM. Pharmacists should routinely provide updates to other healthcare providers on the commonly encountered BBTs as to raise awareness on the potential health complications with their use.

Continuing Medical Education requirements should have a CAM component so that healthcare providers are up to date on health information for established and new products. Though there are several BBT's that are well studied and widely used, their efficacies are variable due to poor regulation and even contamination during the manufacturing process such as lead or mercury. Stricter regulatory mechanisms need to be adhered to by all CAM and BBT's practitioners as this may, over time, lead to a wider acceptance by allopathic and osteopathic physicians alike [20]. Pharmacists need to educate patients regarding the efficacy and potential adverse effects of BBTs in a manner that is consistent with the principles of evidence-based medicine. It is therefore important that pharmacists take an active role in the nutritional assessment and counseling of patients to ensure a healthy lifestyle.

Pharmacists in the U.A.E should educate themselves on the local remedies that are commonly used and should remain vigilant for any symptoms that are not reported to their primary care physicians, as they may be a result of potential drug interactions. Visual assessment of the patient in addition to medication history during a consultation may elucidate potential drug-nutrient or drug-herbal interactions and therefore should be done routinely. Reporting adverse reactions to the local health authority in the U.A.E would result in the safe use of herbal and dietary supplements, raise awareness on serious side effects on the use of a form of BBT, and would solidify the vital role pharmacists have in health education.

\section{Acknowledgement}

We are sincerely grateful to all the participants involved in this project. Specials thanks to : Dr. Ali Akram for his assistance, Faisal Husain and Dr. Muhammad Reza Chaudhry for their advice, and Mr. Harambe for his Dank Web Browsing tips. We would also like to thank our mentor, Dr. Elhadi N. Mahmoud for his guidance and patience throughout the year.

\section{References}

1. (2017) The Use of Complementary and Alternative Medicine in the United States [Internet]. National Center for Complementary and Integrative Health. U.S. Department of Health and Human Services.
2. Waldman SA, Terzic A (2009) Pharmacology and therapeutics principles to practice pp. 1536.

3. Vohra S, Feldman K, Johnston B, Waters K, Boon H (2005) Integrating complementary and alternative medicine into academic medical centers: Experience and perceptions of nine leading centers in North America. BMC Health Services Research 5(1): 78.

4. (2000) Expanding horizons of healthcare: five-year strategic plan 2001 2005. Place of publication not identified: US Department of Health and Human Services. NIH Publication No. 01-5001.

5. Rosenzweig S (2014) Biologically Based Therapies - Special Subjects [Internet]. Merck Manuals Consumer Version.

6. Novella S (2014) Diet Therapy - Special Subjects. Merck Manuals Professional Edition.

7. Ventola CL (2010) Current Issues Regarding Complementary and Alternative Medicine (CAM) in the United States. Part 1: The Widespread Use of CAM and the Need for Better-Informed Health Care Professionals to Provide Patient Counseling. Pharmacy \& Therapeutics 35(8): 461468.

8. Bhadra R, Ravakhah K, Ghosh R (2015) Herb-drug interaction: The importance of communicating with primary care physicians. Australasian Medical Journal 8(10): 315-319.

9. Tarn DM, Guzmán JR, Good JS, Wenger NS, Coulter ID, et al. (2014) Provider and Patient Expectations for Dietary Supplement Discussions. Journal of General Internal Medicine 29(9): 1242-1249.

10. Yetley EA (2007) Multivitamin and multimineral dietary supplements: definitions, characterization, bioavailability, and drug interactions. American Journal of Clinical Nutrition 85(1): 269S-276S.

11. Gardiner P, Phillips R, Shaughnessy AF (2018) Herbal and Dietary Supplement-Drug Interactions in Patients with Chronic Illnesses. Am Fam Physician 77(1): 73-78.

12. Chagan L, Bernstein D, Cheng JW, Harold L Kirschenbaum, Vitalina Rozenfeld, et al. (2005) Use of biological based therapy in patients with cardiovascular diseases in a university-hospital in New York City. BMC Complementary and Alternative Medicine. March 5: 4.

13. Boyer EW (2005) Issues in the Management of Dietary Supplement Use Among Hospitalized Patients. Int J Med Toxicol 1(1): 30-34.

14. US, Department of Health and Human Services. National Institutes of Health. Use and Safety of Dietary Supplements.

15. (2017) US Department of Health and Human Services. FDA U.S. Food and Drug Association. FDA 101: Dietary Supplements.

16. (2017) Help Guide. Dietary Supplements. The Smart and Safe Use of Vitamins and Supplements.

17. Mohammad Y (2009) Drug-Food Interactions and Role of Pharmacist. Asian Journal of Pharmaceutical and Clinical Research 2(4).

18. Mc Hughes M, Timmermann BN (2005) A Review of the Use of CAM Therapy and the Sources of Accurate and Reliable Information. Journal of Managed Care Pharmacy. October 11(8): 695-703.

19. Ventola CL (2010) Current Issues Regarding Complementary and Alternative Medicine (CAM) in the United States. Part 2: Regulatory and Safety Concerns and Proposed Governmental Policy Changes with Respect to Dietary Supplements. Pharmacy \& Therapeutics. September 35(9): 514-522.

20. Kesavadev J, Saboo B, Sadikot S, Das AK, Joshi S et al. (2017) Unproven Therapies for Diabetes and Their Implications. Advances in Therapy 34(1): 60-77. 
ISSN: 2574-1241

DOI: 10.26717/BJSTR.2018.11.002136

Hananeh Abdolrahman R. Biomed J Sci \& Tech Res

(i) This work is licensed under Creative

Commons Attribution 4.0 License

Submission Link: https://biomedres.us/submit-manuscript.php

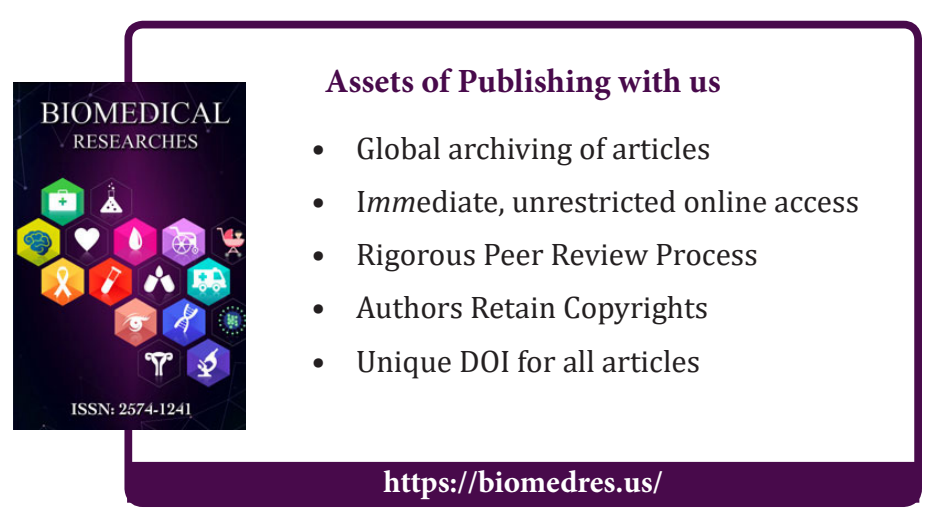

